\title{
Estudio Biométrico de la Inervación de los Músculos Lumbricales de la Mano Humana
}

\author{
Biometric Study of the Innervation of the Lumbrical Muscles of the Human Hand
}

\author{
Cristian Vega ${ }^{1,2}$; Andrés Riveros ${ }^{3,4} \&$ Enrique Olave ${ }^{5}$
}

VEGA, C.; RIVEROS, A. \& OLAVE, E. Estudio biométrico de la inervación de los músculos lumbricales de la mano humana. Int. J. Morphol., 38(4):1096-1105, 2020.

RESUMEN: Los músculos lumbricales (ML) de la mano humana son claves en la propiocepción de la flexoextensión de los dedos. La descripción de su inervación indica que el nervio mediano (NM) inerva los dos ML laterales (L1 y L2) y el nervio ulnar (NU) los ML mediales (L3 y L4). Diversos autores han reportado una gran variabilidad de esta inervación, tanto en los nervios que entregan ramos para estos músculos, como también en la distribución de sus ramos y la presencia de troncos comunes. Por otra parte, el número de ramos que recibe cada ML y los puntos motores $(\mathrm{Pm})$ de los mismos ha sido escasamente reportado. El objetivo de este estudio fue determinar número, ubicación y Pm de los ramos destinados a los ML de la mano humana. Así mismo se estableció el patrón de inervación más frecuente. Para ello se utilizaron 24 manos formalizadas, pertenecientes al laboratorio de Anatomía, de la Universidad Andrés Bello, sede Viña del Mar, Chile. Se realizó una disección convencional por planos de profundidad. En todos los casos, el ramo del músculo L1 se originó del nervio digital palmar propio lateral del dedo índice, de la misma forma, en el $100 \%$ el L2 fue inervado por un ramo del nervio digital palmar común del segundo espacio interóseo. En relación a los ML mediales en un $100 \%$ ambos músculos fueron inervados por ramos del ramo profundo del NU (RPNM). En el caso del L3 en un $92 \%$ se presentó un tronco común con el segundo músculo interóseo palmar, asimismo para L4 existió un tronco común con el tercer músculo interóseo palmar en un $79 \%$. En el $29 \%$, el L3 presentó una inervación dual. Considerando como referencia la línea biestiloidea, los Pm de los ramos del NM fue de 63,96 mm para L1; 67,91 mm para L2 y 68,69 mm para L3. Para los ramos provenientes del RPNU fue de 69,87 mm para L3 y 69, $21 \mathrm{~mm}$ para L4. Los resultados obtenidos aportan al conocimiento anatómico de la inervación de los músculos lumbricales y es de utilidad en procedimientos de neurocirugía que busquen la restauración de la funcionalidad de la mano.

PALABRAS CLAVE: Anatomía; Inervación; Músculos Lumbricales; Nervio Mediano; Ramo Profundo del Nervio Ulnar; Ramo Comunicante; Variaciones Anatómicas.

\section{INTRODUCCIÓN}

La gran destreza y la versatilidad de la mano se debe en gran medida a sus músculos intrínsecos, de éstos, los músculos lumbricales son cuatro pequeños músculos ubicados en la región palmar central de la mano (Mehta \& Gardner, 1961). Dispuestos de forma anexa a los tendones del músculo flexor profundo de los dedos, estos músculos colaboran con la flexión de la articulación metacarpofalángica (MCF), y producto de su inserción en la expansión extensora, complementan la extensión de las articulaciones interfalángicas proximal y distal. En este sentido, Palti \& Vigler (2012), señalaron que la labor principal de estos músculos está asociada principalmente a la extensión de las articulaciones interfalángicas. Lo anterior sería facilitado por- que estos músculos lumbricales disminuyen el torque en la flexión de la articulación MCF que realiza el músculo flexor profundo de los dedos.

Nombrados por la semejanza a la lombriz de tierra (Lumbricus terrestris), estos músculos presentan un rol clave en las actividades propioceptivas de los movimientos de los últimos cuatro dedos. Lo anterior se debería a la gran cantidad de mecanorreceptores, principalmente husos neuromusculares; por ello, la contracción del músculo flexor profundo de los dedos genera la elongación de los músculos lumbricales, lo cual influye en la inhibición de los músculos extensores de los dedos. Por esta razón, a los músculos

\footnotetext{
${ }^{1}$ Departamento de Morfología, Facultad de Medicina, Universidad Andrés Bello, 2531015, Viña del Mar, Chile.

${ }^{2}$ Programa de Magíster en Ciencias, Mención Morfología, Facultad de Medicina, Universidad de La Frontera, Temuco, Chile.

${ }^{3}$ Departamento de Ciencias Morfológicas, Facultad de Medicina y Ciencia, Universidad San Sebastián, Lientur 1457, Concepción 4080871, Chile.

${ }^{4}$ Programa de Doctorado en Ciencias Morfológicas, Universidad de La Frontera, Temuco, Chile.

${ }^{5}$ Facultad de Medicina, Universidad de La Frontera, Temuco, Chile.
} 
lumbricales se les asigna una labor clave en los movimientos rápidos y alternos de los dedos de la mano como escribir y tocar instrumentos (Rabischong, 1971; Palti \& Vigler).

La descripción clásica respecto de la inervación de estos músculos indica que los lumbricales laterales (L1 y L2) están inervados por ramos originados desde el nervio digital palmar propio lateral del dedo índice (DPPLi) y el nervio digital palmar común (DPC) del II espacio interóseo, respectivamente. En ambos casos, estos nervios se originan de la distribución que el nervio mediano (NM) presenta en la mano. En el caso de los dos músculos lumbricales mediales (L3 y L4), sus ramos surgen directamente desde el ramo profundo del nervio ulnar (RPNU) o desde troncos comunes que además de entregar ramos para estos músculos lumbricales, otorgan ramos para los músculos interóseos dorsales (ID) e interóseos palmares (IP) (Testut \& Latarjet, 1972; Rouvière \& Delmas, 2005; Llusá et al., 2013; Standring, 2016).

En la búsqueda de datos más específicos, Afroze et al. (2014), estudiaron 54 manos estableciendo que el patrón normal se presentó en el $85 \%$ de los casos. Dentro de las variaciones de este estudio, destacó que en un 5,5\% el L2 estuvo inervado por ramos provenientes del nervio ulnar (NU). En la vereda opuesta, Hur (2017), en un estudio que incluyó 100 manos, reportó que la presentación clásica se presentó en el $26 \%$. En este sentido Wynter \& Dissabandara (2017), realizaron una revisión que incluyó 70 artículos que analizaban la inervación de los músculos de la mano. En dicha investigación, relataron que el patrón clásico de inervación de la mano se encuentra aproximadamente en un tercio de la población y un quinto de la misma presenta variaciones importantes. De todas formas, existiría una baja probabilidad de que todos los músculos intrínsecos de la mano sean inervados por un solo nervio, sea este el NM o el NU. Estas variaciones de inervación estarían asociadas a intercambio de paquetes axonales entre los NU y NM en el antebrazo o mano (Tountas \& Bergmann, 1993).

Si bien la literatura es consistente al momento de referirse al origen de los ramos para el L1 y L2, en lo que refiere al origen de los ramos destinados al L3 y L4 se describen frecuentemente la presencia de troncos comunes. Al respecto Kaplan (1961), indicó que para el caso de los músculos L3 y L4 el origen de estos ramos es independiente de los otros ramos que el RPNU entrega a los músculos IP e ID. Por otra parte, Olave \& del Sol (2008), reportaron que desde el RPNU se originaron troncos comunes que además de generar ramos para los músculos L3 y L4 se presentaron otros destinados a los músculos 2do y 3er IP. Esta descripción también reportada por Tubbs et al. (2015) y Homma \& Sakai (1992), describieron la existencia de un patrón regular que incluyó la presencia de dos troncos comunes en el segundo, tercer y cuarto espacio intermetacarpiano. De ellos, el tronco medial de cada espacio originó ramos destinados tanto a los músculos L3 y L4 como también a los IP e ID.

En el caso específico del músculo L3 existen reportes diversos. Autores como Tountas \& Bergmann y Standring señalaron que este músculo es inervado a menudo por ramos provenientes de los NM y NU. Al respecto Sunderland $\&$ Ray (1946), en una muestra de 20 manos, reportaron que en un $25 \%$ de los casos el L3 estuvo inervado en forma exclusiva por ramos originados del NM, que en la mayoría de los casos aportó con un solo ramo. En esta misma línea, Hur estableció que en un $60 \%$ el L3 estuvo inervado tanto por ramos del NM como del NU. Así mismo, relató que la inervación exclusiva del L3 por parte del NM se asoció a la presencia de un ramo comunicante entre los nervios digitales palmares comunes que nacen de los NM y NU. En dicho escenario, algunas fibras del NU podrían formar parte del ramo que el NM le entrega al L3. Esta descripción coindice con las descripciones de Mehta \& Gardner, que relataron la existencia de un asa nerviosa de la cual surgen ramos para el L3. En la búsqueda de la correcta denominación de este ramo comunicante, Riveros et al. (2019) reportaron que si bien su denominación clásica ha sido ramo comunicante de Berretini, su correcto término sería ramus communicans cum nervo digitali palmari communi.

Si bien es conocido que los músculos L1 y L2 reciben sus ramos por la cara palmar y cercano a su margen lateral, y que al contrario, los lumbricales L3 y L4 lo hacen por dorsal y próximo a su margen medial, el número de ramos y puntos motores (Pm) están escasamente reportados. Respecto al número de ramos, Testut \& Latarjet señalaron que el músculo L2 recibe un solo ramo, sin embargo, Sunderland \& Ray informaron que en el $15 \%$ de su muestra, el L1 recibió dos ramos y en un $10 \%$ tres. En el caso del músculo L2, solo un $4 \%$ recibió dos ramos. Esta descripción fue reafirmada por Kaplan, quien señaló que estos lumbricales laterales reciben uno o dos ramos cada uno. Al respecto, Lauritzen et al. (1996), en un estudio que incluyó nueve manos, describieron que en el $44 \%$ de los casos, los músculos lumbricales presentaron un ramo cada uno, señalando que en el porcentaje restante estos músculos recibieron dos o más ramos.

En relación a los Pm, en los músculos L1 y L2, sus ramos se localizan en su tercio medio (Kaplan; Tountas \& Bergmann). En este sentido, Lauritzen et al. concluyeron que la inervación de los diferentes músculos lumbricales ocurre típicamente en los tercios proximal y medio. En la misma línea, Hughes \& Clarke (1995), describieron la arborización del RPNU y los Pm de los músculos L3, L4, ID e IP, usando como referencia la línea biestiloidea (LBEst). De esta forma, reportaron la relación entre la ubicación del 
Pm con el metacarpiano respectivo, señalando que el Pm del L3 se ubicó a nivel del tercio medio del tercer metacarpiano y que el Pm del L4 lo hizo a nivel del tercio distal del cuarto metacarpiano.

Basado en lo anterior, el propósito del presente estudio fue determinar el origen, Pm y biometría de los ramos destinados a los músculos lumbricales de la mano humana. Lo anterior contribuirá en profundizar el conocimiento respecto de la inervación de los músculos de la mano, lo que sin duda, será de importancia para la anatomía quirúrgica del miembro superior.

\section{MATERIAL Y MÉTODO}

En el presente estudio se utilizaron 24 miembros superiores de individuos adultos ( 6 derechos y 18 izquierdos) pertenecientes al Departamento de Morfología de la Facultad de Medicina de la Universidad Andrés Bello, Viña del Mar, Chile. Estos cadáveres se encontraron fijados en una solución con formaldehído al $10 \%$ y almacenados en cámara de frío a $4{ }^{\circ} \mathrm{C}$. Las muestras utilizadas no presentaban patologías macroscópicas ni disecciones previas.

Esta investigación de carácter cuantitativo, no experimental, transeccional y descriptiva utilizó una metodología de disección por planos. Dicha disección se realizó tanto en las regiones palmar lateral, central y medial como también en el compartimento anterior del antebrazo, utilizando para ello material quirúrgico ad hoc.

Esta disección se inició trazando inicialmente una línea vertical en la región palmar central de la mano, desde el pliegue distal de la muñeca (PDM) hasta la cabeza del tercer metacarpiano. A la altura de esta cabeza se realizó una incisión transversa distal que permitió el normal retiro del tejido cutáneo. Después de que la piel y el tejido subcutáneo de la palma fueron removidos, se identificó la aponeurosis palmar, la cual se retiró desde distal hacia proximal, logrando exponer el retináculo flexor, los nervios digitales palmares comunes provenientes del NM y los ramos destinados a los vientres de los músculos L1 y L2. Posterior a la medición y registro de los ramos destinados a estos músculos, se realizó la disección profunda que permitió exponer los ramos motores que el RPNU destinó a los músculos L3 y L4. Para el desarrollo de esta disección profunda fue necesario ampliar el trabajo anatómico hacia la región antebraquial anterior, la que permitió exponer los tendones de los músculos flexor superficial y profundo de los dedos. Dichos tendones fueron seccionados horizontalmente a nivel del tercio distal del antebrazo y se traccionaron hacia distal, lo que permitió acceder al plano profundo de la celda palmar media. Posterior a un delicado retiro del panículo adiposo presente en esta zona, se visualizó, midió y registró la distribución del RPNU a los grupos musculares anteriormente mencionados.

Respecto de los ramos observados, se estableció su origen nervioso, indicando si correspondió a un ramo aislado o surgió desde un tronco común. Junto con medir la longitud de cada ramo y considerando como referencias el PDM y la LBEst, se midió la distancia de los diferentes puntos de origen nervioso y los Pm, definiendo a estos últimos como el punto de ingreso de estos ramos a los vientres musculares correspondientes. Complementariamente, también fueron registrados estos Pm según el tercio muscular en el cual penetraban en cada músculo lumbrical; para ello se registró la longitud total de cada músculo.

Las mediciones de las estructuras involucradas en el estudio se realizaron con un caliper digital Mitutoyo ${ }^{\circledR}$ de $0,01 \mathrm{~mm}$ de precisión. Complementariamente las muestras disecadas fueron fotografiadas con una cámara digital Canon ${ }^{\circledR}$ Rebel XTI y se realizó un esquema que consideró la distribución de los ramos analizados.

\section{RESULTADOS}

En la totalidad de las 24 manos analizadas se presentaron los cuatro músculos lumbricales, no existiendo variaciones anatómicas en la forma y ubicación de estos músculos. La longitud promedio de cada músculo, junto a las longitudes máxima y mínima se encuentran resumidas en la Tabla I. Así mismo, las longitudes de los ramos nerviosos analizados junto a las longitudes máxima y mínima se encuentran resumidas en la Tabla II.

Tabla I. Longitud de los músculos lumbricales de la mano.

\begin{tabular}{ccccc}
\hline Músculo & $\begin{array}{c}\text { Longitud promedio } \\
(\mathrm{mm})\end{array}$ & D.E & $\begin{array}{c}\text { Longitud mínima } \\
(\mathrm{mm})\end{array}$ & $\begin{array}{c}\text { Longitud máxima } \\
(\mathrm{mm})\end{array}$ \\
\hline M. lumbrical 1 & 50,00 & 9,12 & 23,04 & 74,02 \\
M. lumbrical 2 & 43,64 & 6,22 & 36,25 & 58,73 \\
M. lumbrical 3 & 41,99 & 8,46 & 26,08 & 62,37 \\
M. lumbrical 4 & 33,11 & 6,79 & 17,33 & 46,63 \\
\hline
\end{tabular}


Tabla II. Longitud de los ramos motores que inervan los músculos lumbricales.

\begin{tabular}{lcccc}
\hline Ramo & Longitud $(\mathrm{mm})$ & D.E & Mínimo $(\mathrm{mm})$ & Máximo $(\mathrm{mm})$ \\
\hline M. lumbrical 1 & 6,48 & 2,38 & 3,11 & 12,58 \\
M. lumbrical 2 & 8,17 & 3,66 & 3,81 & 19,53 \\
M. lumbrical 3* & 8,02 & 4,05 & 4,02 & 15,91 \\
M. lumbrical 3** & 25,25 & 4,43 & 15,69 & 34,20 \\
M. lumbrical 4 & 29,53 & 4,20 & 21,89 & 37,56 \\
\hline * Ramos provenientes del nervio mediano. **Ramos provenientes del nervio ulnar.
\end{tabular}

En relación al número de ramos que recibió cada músculo lumbrical, en el total de las manos analizadas el L1 recibió un solo ramo (R1L1), situación que en el caso del L2 alcanzó el $96 \%$ (R1L2) de los casos. En este sentido sólo una muestra presentó dos ramos destinados al L2 (R1L2, R2L2). En el caso de los lumbricales mediales, en el $71 \%$ de los casos, el músculo L3 recibió un solo ramo, mientras que en el $25 \%$ recibió dos ramos. Existió una muestra $(4 \%)$ en la cual este lumbrical recibió tres ramos. En esta misma línea, en el $100 \%$ de las muestras el músculo L4 recibió un ramo.

Respecto a los ramos secundarios que nacen de los ramos primarios del NM, 3 muestras presentaron ramos secundarios en relación con L1 (13\%), de éstos, dos casos presentaron 2 ramos secundarios y uno presentó 3 . Con respecto a los ramos motores destinados al L2, una muestra presentó tres ramos secundarios $(4 \%)$.
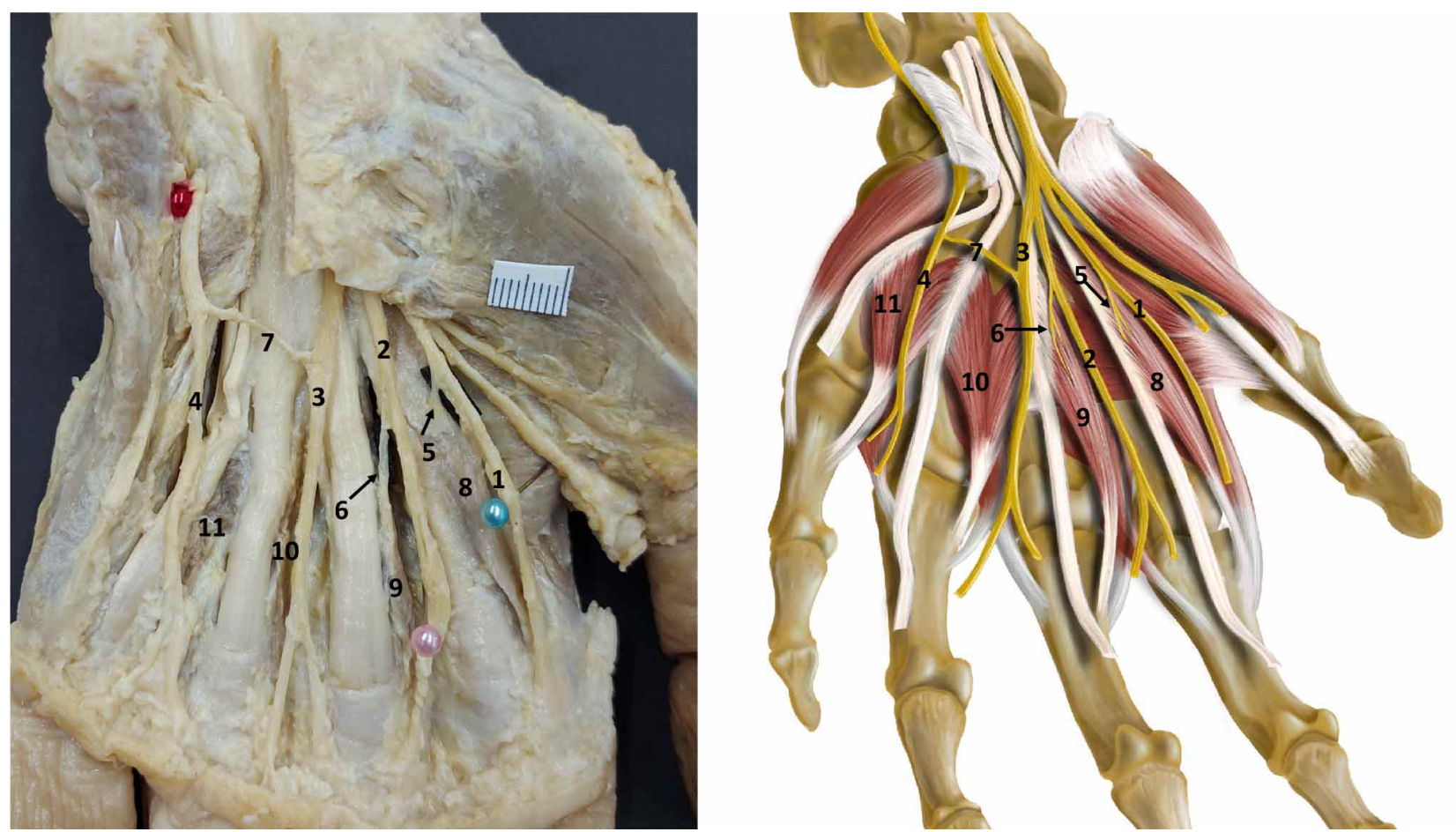

Fig. 1. Mano izquierda. 1: Nervio DPP Li, 2: Nervio DPC del segundo espacio interóseo, 3: Nervio DPC del tercer espacio interóseo, 4: Nervio DPC del cuarto espacio interóseo, 5: Ramo motor para el músculo L1, 6: Ramo motor para el músculo L2, 7: Ramo comunicante, 8: Músculo L1, 9: Músculo L2, 10: Músculo L3, 11: Músculo L4. 

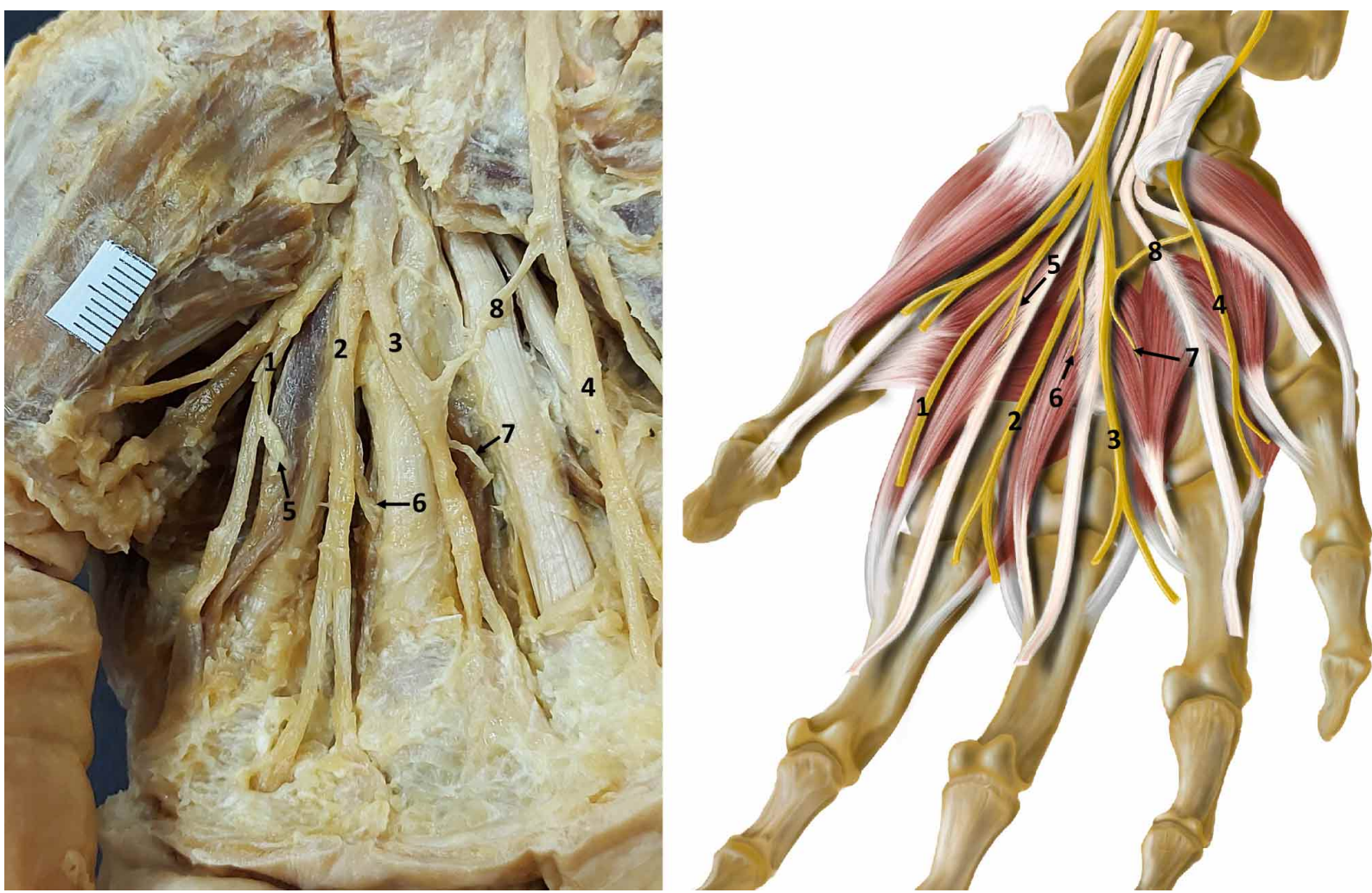

Fig. 2. Mano derecha. 1: Nervio DPP Li, 2: Nervio DPC del segundo espacio interóseo, 3: Nervio DPC del tercer espacio interóseo, 4: Nervio DPC del cuarto espacio interóseo, 5: Ramo motor para el músculo L1, 6: Ramo motor para el músculo L2, 7: Ramo Motor para el músculo L3 8: Ramo comunicante entre los nervios DPC del tercer y cuarto espacio interóseo.
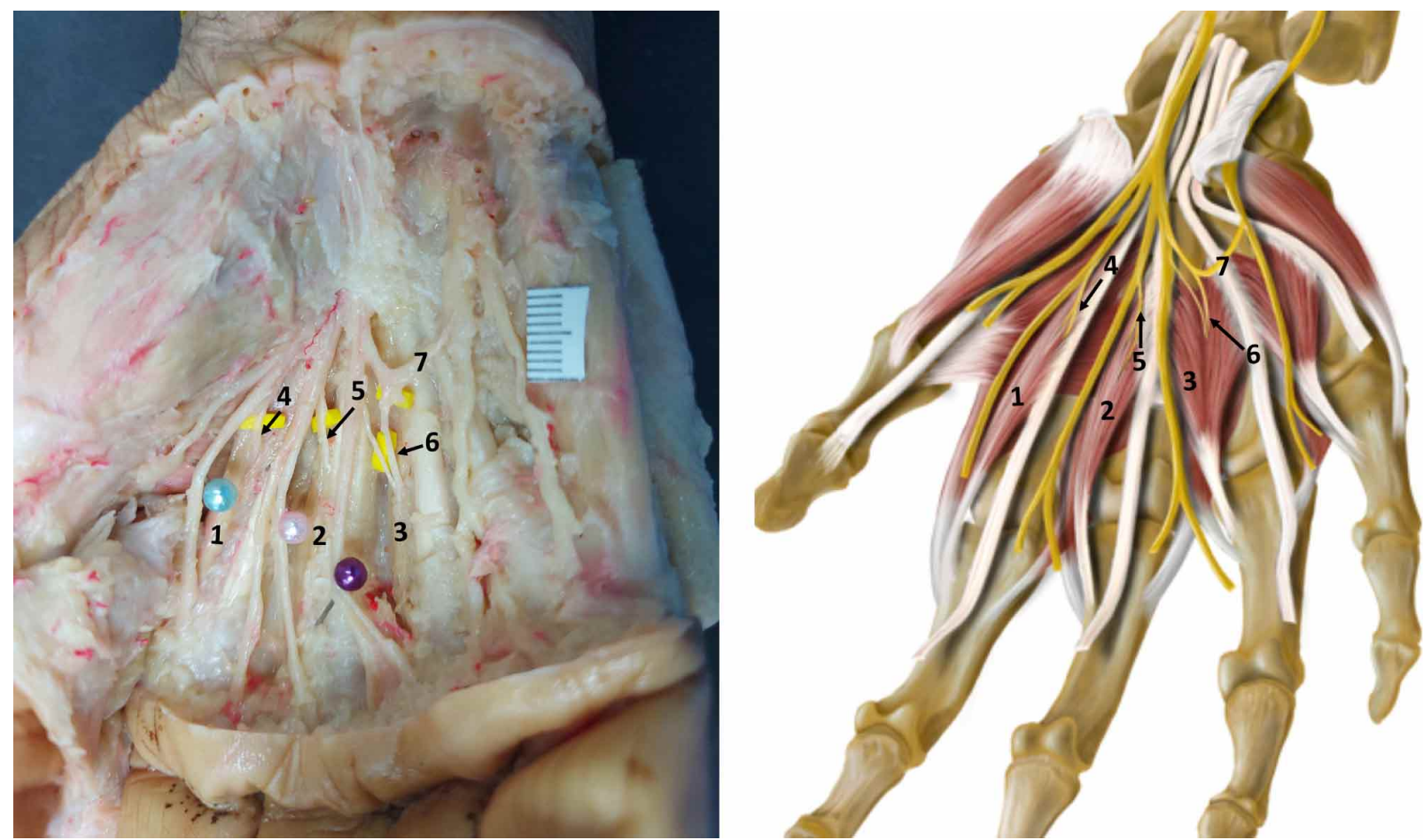

Fig. 3. Mano derecha. 1: Músculo L1, 2: Músculo L2, 3: Músculo L3, 4: Ramo motor para el músculo L1, 5: Ramo motor para el músculo L2, 6: Ramo Motor para el músculo L3 con contribución del nervio DPC del tercer espacio interóseo y el ramo comunicante. 7: Ramo comunicante entre los nervios DPC del tercer y cuarto espacio interóseo. 
En relación a lo anterior, la presencia del ramo comunicante descrito se observó en el $42 \% \%$ de los casos (10 muestras), con un promedio de $14,9 \mathrm{~mm}$ de longitud, los cuales presentaron una dirección oblicua, de proximal a distal, uniendo en todos los casos el nervio DPC del IV espacio interóseo con el nervio DPC del III espacio interóseo (Figs. 1 y 2).

$\mathrm{Al}$ analizar los ramos destinados a los lumbricales mediales, en el $100 \%$ de los casos el RPNU otorgó ramos a estos músculos, ya fuera como un ramo único o formando un tronco común para la inervación de los músculos oponente del dedo mínimo y/o IP (Fig. 4). En 22 casos (92\%) y a nivel del $3^{\text {er }}$ espacio intermetacarpiano, el RPNU dio origen a un tronco común que otorgó ramos a los músculos L3 e IP2. En los dos casos restantes (8\%), se presentó un ramo único y directo hacia este músculo lumbrical. En el caso del

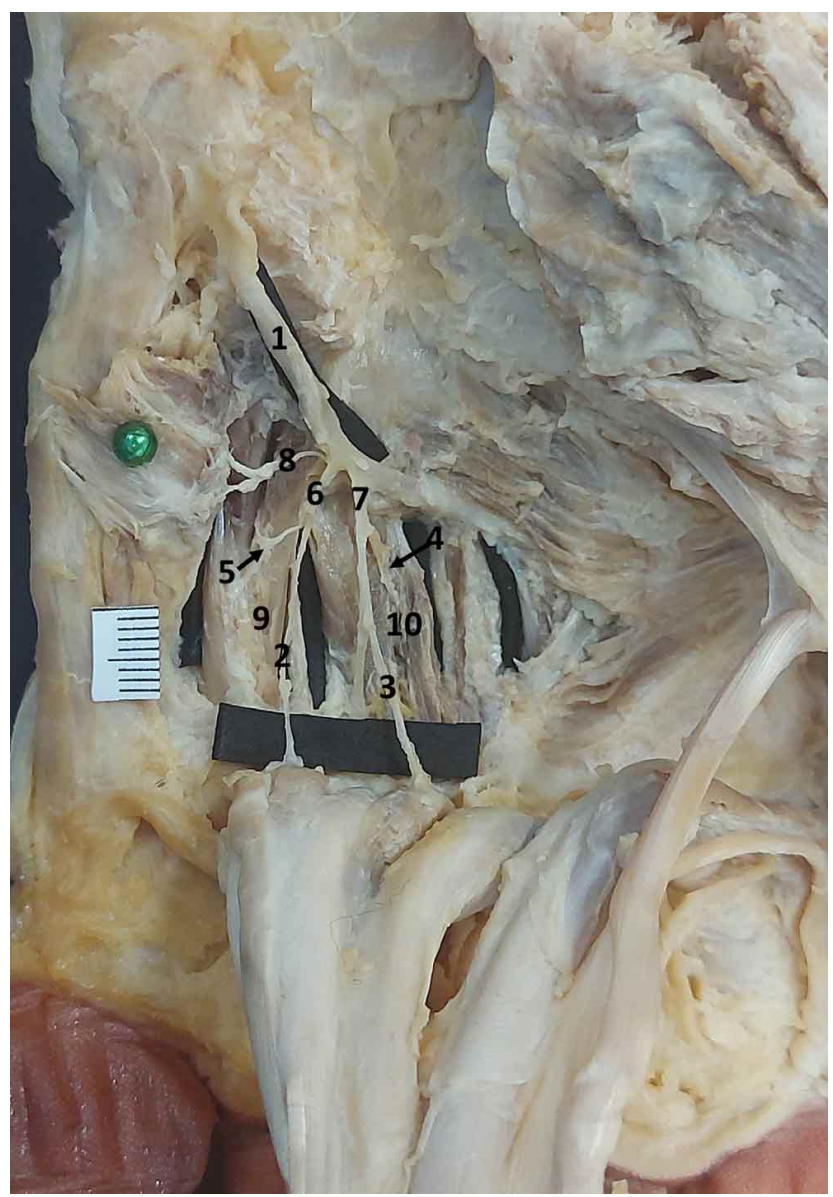

Fig. 4. Mano izquierda. 1: Ramo profundo del nervio ulnar, 2: Ramo motor del músculo L4, 3: Ramo motor del músculo L3, 4: Ramo motor del segundo músculo interóseo palmar, 5: Ramo motor del tercer músculo interóseo palmar, 6: Tronco común para el tercer músculo interóseo palmar y el músculo L4, 7: Tronco común para el segundo músculo interóseo palmar y el músculo L3, 8: Ramo motor para el músculo oponente del dedo mínimo, 9: Tercer músculo interóseo palmar 10: Segundo músculo interóseo palmar. músculo L4, en 19 de las muestras (79 \%) se presentó un tronco común que entregó ramos para este lumbrical y para el músculo IP3. Complementariamente, en tres casos (13 $\%$ ) se identificó un tronco común que otorgó ramos para los músculos L4, IP3 y oponente del meñique (8\%). Asimismo en dos casos ( $8 \%$ ) este músculo L4 fue inervado a través de un ramo único (Fig. 5).

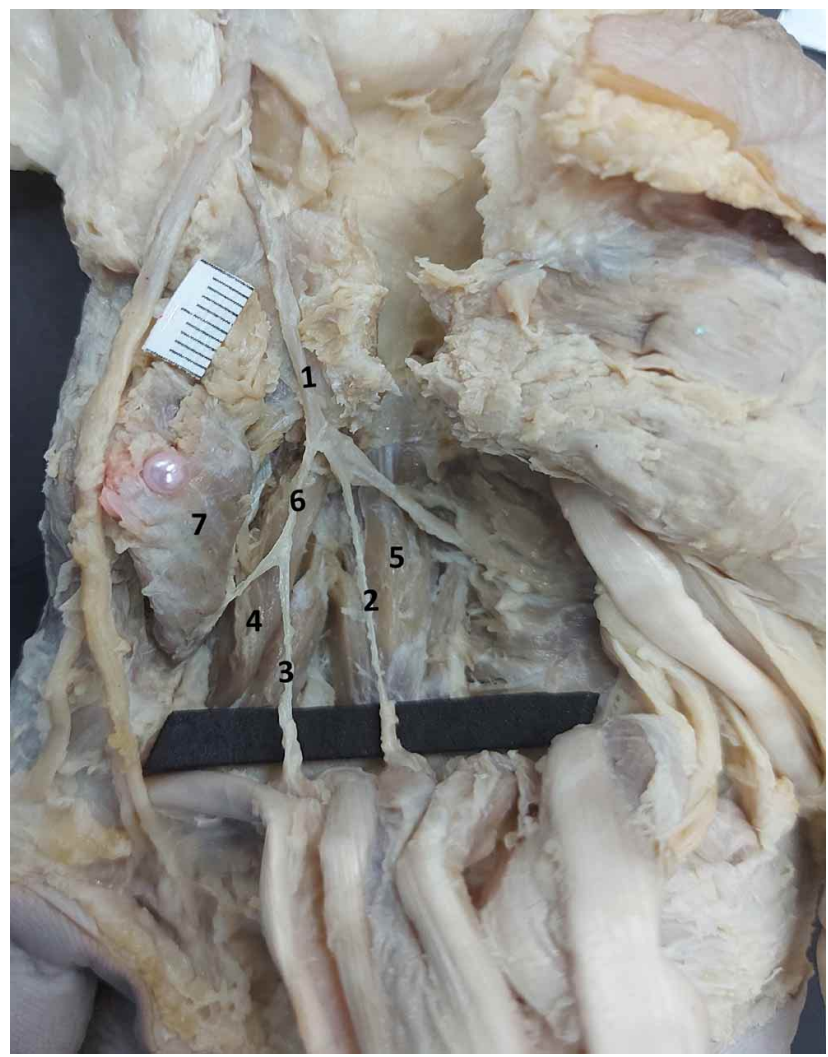

Fig. 5. Mano izquierda. 1: Ramo profundo del nervio ulnar, 2: Ramo motor del músculo L3, 3: Ramo motor del músculo L4, 4: Tercer músculo interóseo palmar, 5: Segundo músculo interóseo palmar, 6: Tronco común para los músculos L4, tercer interóseo palmar y oponente del dedo mínimo.

Cabe destacar que en relación al origen y forma de presentación de los ramos analizados (ramo independiente o desde un tronco común), en el $71 \%$ de la muestra se observó el patrón de descripción clásico, el cual consideró que los músculos L1 y L2 fueron inervados por ramos originados de nervios que surgieron del nervio mediano. Asimismo, los músculos L3 y L4 fueron inervados por ramos originados del RPNU. En el caso del $29 \%$ restante, la inervación del músculo L3 fue dual, ya que junto con recibir ramos del RPNU también recibió ramos del nervio DPC del III espacio interóseo o del ramo comunicante antes descrito.

Al profundizar en la descripción de los casos que presentaron el patrón clásico, encontramos que en el $65 \%$ de 
éstos, la inervación de los músculos L3 y L4 fue otorgada por troncos comunes que, originados del RPNU, tambien entregaron ramos para los músculos IP2 e IP3, respectivamente.

En relación al punto de origen de los nervios motores primarios que nacen del NM, la distancia promedio entre el PDM y el músculo L1 fue de 48,1 mm; para músculo L2 fue de 51,9 mm y para el músculo L3 56,8 mm. Para el punto de origen de los nervios motores primarios que nacen del nervio ulnar, la distancia promedio entre el PDM y L3 fue de 40,2 mm y para L4 fue de 36,8 $\mathrm{mm}$. Los datos respecto al promedio de la distancia del punto de origen de cada ramo, junto a las distancias máxima y mínima se encuentran resumidas en la Tabla III.

Respecto a los Pm de los músculos lumbricales y considerando la LBEst como referencia; las distancias promedio entre el Pm y la LBEst fue de 63,96 mm \pm 7,55 mm para el músculo L1 y 67,91 \6,41 mm para el L2. En esta misma línea, las distancias promedio para los músculos L3 y L4 alcanzaron $69,87 \pm 6,36 \mathrm{~mm}$ y $69,21 \pm 5,96 \mathrm{~mm}$, respectivamente. Caso aparte fueron los siete casos en la cual el
NM (a través del nervio DPC del III espacio interóseo o de un ramo comunicante) entregó ramos para el L3. Estos presentaron Pm que promediaron 68,69 \pm 3,05 mm. Los datos respecto al promedio de la distancia de los Pm de cada ramo, junto a las distancias máxima y mínima se encuentran resumidas en la Tabla IV.

Al analizar los Pm según el tercio del vientre muscular de cada músculo lumbrical, el músculo L1 los presentó en el tercio medio en $79 \%$ y en su tercio proximal en un 21 $\%$. En el caso del músculo L2 sus Pm se distribuyeron en el tercio medio (71\%), en el tercio proximal $(25 \%)$ y en el distal (4\%). Aplicada esta misma metodología a los músculos lumbricales mediales, en el músculo L3 los Pm se localizaron en su tercio distal $(87 \%)$ y en el tercio medio (13 $\%$ ). Para el caso del músculo L4, en el $83 \%$ de los casos los Pm se ubicaron en el tercio distal y en $17 \%$ se presentaron en el tercio medio de este vientre muscular.

Para los siete casos en la cual existieron ramos para el músculo L3, originados desde ramos del NM, los Pm se ubicaron en el tercio medio en un $43 \%$ y en el tercio distal en un $57 \%$.

Tabla III. Distancia entre el pliegue distal de la muñeca y el origen de los ramos musculares.

\begin{tabular}{lcccc}
\hline \multicolumn{1}{c}{ Músculo } & Distancia promedio $(\mathrm{mm})$ & D. E. & Distancia mínima $(\mathrm{mm})$ & Distancia máxima $(\mathrm{mm})$ \\
\hline M. lumbrical 1 & 48,07 & 5,79 & 37,52 & 59,94 \\
M. lumbrical 2 & 51,95 & 5,78 & 37,85 & 69,40 \\
M. lumbrical 3* & 56,84 & 11,23 & 47,83 & 81,11 \\
M. lumbrical 3** & 40,20 & 4,47 & 31,94 & 50,01 \\
M. lumbrical 4 & 36,84 & 4,52 & 29,69 & 47,06 \\
\hline
\end{tabular}

* Ramos provenientes del nervio mediano. **Ramos provenientes del nervio ulnar.

Tabla IV. Distancia entre la Línea Biestiloídea y los puntos motores.

\begin{tabular}{lcccc}
\hline \multicolumn{1}{c}{ Músculo } & Distancia promedio $(\mathrm{mm})$ & D.E. & Distancia mínima $(\mathrm{mm})$ & Distancia máxima $(\mathrm{mm})$ \\
\hline M. lumbrical 1 & 63,96 & 7,55 & 49,05 & 82,22 \\
M. lumbrical 2 & 67,91 & 6,41 & 55,33 & 79,78 \\
M. lumbrical 3 & 68,69 & 3,05 & 65,01 & 71,76 \\
M. lumbrical 3** & 69,87 & 6,36 & 55,03 & 87,71 \\
M. lumbrical 4 & 69,21 & 5,96 & 57,02 & 84,12 \\
\hline
\end{tabular}

* Ramos provenientes del nervio mediano. **Ramos provenientes del nervio ulnar.

\section{DISCUSIÓN}

Las notables funciones que desarrollan los $\mathrm{Mm}$. lumbricales en las actividades de la mano obligan a los cirujanos a preservar la inervación de estos músculos en cualquier intervención quirúrgica. Para ello es indispensable conocer las principales variaciones de inervación de los mismos.

En relación al número de ramos que recibió cada músculo lumbrical, nuestros hallazgos coinciden con las descripciones de Testut \& Latarjet, Kaplan y Olave \& del Sol, quie- nes informaron de la presencia de un ramo para cada músculo lumbrical. Si bien en el $4 \%$ de nuestra muestra el L2 recibió dos ramos, dicha cifra es notoriamente inferior al $44 \%$ descrito por Lauritzen et al., quienes además concluyeron que los músculos L2 y L4 pueden frecuentemente presentar múltiples ramos. De esta forma, y en el escenario de que se produzcan traumatismos u otras patologías que afecten estos únicos ramos, la importante actividad de estos músculos podría estar comprometida. 
$\mathrm{Al}$ analizar el origen de estos ramos y considerando que en el $71 \%$ de nuestra serie se presentó el patrón clásico, cifra que se acerca al $85 \%$ que reportó Afroze et al., pero es inferior a los descrito por Mehta \& Gardner que reportaron este patrón en un $92 \%$ de su serie. En este sentido diferimos con lo informado por Hur, quien estableció que el patrón clásico fue observado en el $26 \%$ de los casos, siendo el de mayor frecuencia $(60 \%)$ aquel en donde el NM inervó los músculos L1 y L2 y el NU inervó al L4. A lo anterior se sumó una inervación dual del L3; cifra que en nuestro estudio alcanzó un $29 \%$. En este sentido Wynter \& Dissabandara señalaron que el escenario de una inervación dual varía entre un 7 y un $64 \%$ de los casos, rango que incluye tanto el estudio de Hur como el nuestro. En esta misma línea y considerando que la inervación del músculo L3 en todos los casos incluyó ramos originados directamente del RPNU o desde un tronco común, nuestra investigación no presentó casos en la cual este músculo recibiera ramos exclusivamente del NM o algunos de sus ramos, situación que Sunderland \& Ray reportaron en el $25 \%$ de su muestra.

Por otra parte, es importante considerar que en el $100 \%$ de nuestros casos analizados, el músculo L1 estuvo inervado por el nervio DPPLi y el L2 recibió sus ramos desde el nervio DPC del II espacio interóseo, descripción que coincide con la mayoría de los autores citados. En este sentido, si consideramos el planteamiento de Palti \& Vigler de que la inervación de los dos lumbricales laterales es originada desde nervios definidos como nervios digitales palmares; tal denominación podría conducir a errores que en procedimientos quirúrgicos, particularmente en cirugías de movilización de nervios cutáneos, en la cual se podría generar la denervación de estos músculos.

Cabe mencionar que nuestra investigación no presentó casos en la cual el músculo L2 fuera inervado por ramos del RPNU. Así mismo, tampoco registramos casos en la cual el NM inervó los tres lumbricales laterales y el NU el lumbrical restante, situación que fue reportada por Hur en el $6 \%$ de su serie.

En esta misma línea, nuestro estudio no exhibió casos con inervación total de los músculos lumbricales por parte de un solo nervio, a pesar de que Rowntree (1949), indicó que un $20 \%$ de su muestra se presentaron variaciones que incluyeron inervación total de la mano por parte del NU o la inervación de los músculos interóseos por parte del nervio mediano. Por ello, coincidimos con Tountas \& Bergmann en su planteamiento de que existe una baja probabilidad de que todos los músculos intrínsecos de la mano estén inervados por un solo nervio.
Respecto de la presencia del ramo comunicante entre los nervios DPC del III y IV espacio interóseo, Ferrari \& Gilbert (1991) y Olave et al. (1998), reportaron que en el $90 \%$ y $96 \%$ de los casos, respectivamente, se presentó este ramo de comunicación, el cual en su mayoría se dispuso de forma oblicua originándose desde el nervio DPC del IV espacio interóseo (originado desde la división superficial del NU) e ingresando en el epineuro del nervio DPC del III espacio interóseo (originado del NM). Si bien coincidimos con la descripción de disposición y trayecto de este ramo comunicante, la cifra es altamente superior a nuestro $42 \%$. En este sentido, para Ferrari \& Gilbert las fibras de esta comunicación estarían destinadas al territorio cutáneo presente en los márgenes adyacentes de los dedos medio y anular, en cambio Hur concluyó que el destino de este grupo axonal sería la inervación del músculo L3, agregando que en el $34 \%$ de sus casos, este músculo estuvo inervado por ramos del nervio DPC del III espacio interóseo que surgieron distal al ramo comunicante, situación que se presentó en dos de nuestros casos. Sumado a ello, Mehta \& Gardner reportaron que un 19,7\% de los casos se presentó un ramo adicional desde un asa que se formó entre el ramo superficial del NU y el nervio DPC del III espacio interóseo. Esta particular variación anatómica en nuestro estudio estuvo presente en una sola muestra (4\%), la cual también incluyó un ramo adicional independiente proveniente del nervio DPC del III espacio interóseo (Fig. 3).

En este sentido y tal como lo relataron Olave et al., esta descripción debe ser considerada en cirugías de liberación del canal del carpo, ya que producto de la incisión que se realiza para seccionar el retináculo flexor existiría la posibilidad de dañar este ramo comunicante. Lo anterior podría generar eventuales secuelas motoras sobre el músculo L3 o déficit sensitivo en la región cutánea comprendida entre el margen medial del dedo medio y el margen lateral del dedo anular.

En esta misma línea también compartimos la conclusión de Hur, quien señaló que se esperaría que en el caso de presentar lesiones por atrapamiento o traumatismos, las secuelas de éstas serían diferentes bajo la presencia de un ramo comunicante entre los NM y NU.

En relación a la forma de presentación de los ramos destinados a los músculos lumbricales, claramente existen diferencias si comparamos los lumbricales laterales versus los mediales. En este sentido, nos sumamos a autores como Kaplan, Testut \& Latarjet, Tountas \& Bergmann, Lauritzen et al. y Llusá et al. que relataron que los ramos para los músculos L1 y L2 surgen de forma aislada o independiente, situación que observamos en la totalidad de nuestros casos. 
Por otra parte, la inervación de los músculos L3 y L4 presenta descripciones contradictorias, por una parte, Kaplan señaló que estos músculos reciben un ramo independiente desde el RPNU, sin embargo, esa forma de presentación solo la observamos en 4 muestras, de las cuales, en dos, el RPNU inervó directamente el músculo L3 y en las otras dos, se repitió este formato en la inervación del músculo L4. Al contrario, autores como Olave \& del Sol, señalaron que el músculo L4 en un $90 \%$ recibió un ramo proveniente de un tronco común con el músculo IP3. Misma situación se presentó al inervar el L3 ya que en el $70 \%$ se presentó un tronco común con el músculo IP2. En este sentido, nuestra investigación coincide con lo descrito por estos últimos autores, tanto en la descripción como en la cifra, ya que en el 92 $\%$ de nuestro estudio existió un tronco común que otorgó ramos a los músculos L3 e IP2. De la misma forma en el 79 $\%$ de los casos se presentó un tronco común que entregó ramos para los músculos L4 e IP3.

Con relación a lo anterior, otra descripción común es lo que reportaron Gil et al. (2016), en la cual, en estudio de 36 manos se describió la presencia constante de troncos que surgieron desde el RPNU. En concreto, en el $100 \%$ de su muestra se presentó un tronco común que entregó ramos para los músculos oponente del dedo mínimo, IP3 y L4. En esta misma línea Atkins et al. (2009), describieron que el tronco común está presente en el tercer espacio interóseo, no así en el 4to espacio. De estas dos descripciones, nuestro estudio coincide con lo descrito por Gil et al., sin embargo discrepamos en la cifra informada, ya que si bien se presentaron casos en la cual un tronco común otorgó ramos para los músculos L4, IP3 y oponente del dedo mínimo, esto solamente se observó en tres casos (13\%).

Finalmente, al analizar los Pm de los músculos lumbricales, Sunderland \& Ray informaron que desde el proceso estiloides del radio, el músculo L1 presentó su Pm a una distancia que fluctuó entre los 55 y los $98 \mathrm{~mm}$, cifra que para el músculo L2 alcanzó un intervalo de 54 a $95 \mathrm{~mm}$. En los casos en la cual el NM le otorgó ramos para el L3 estos presentaron un Pm que varió entre los 70 a los 100 mm. En la misma línea, Sunderland \& Hughes (1946), informaron que el Pm del músculo L3 se dispuso entre los 57 a los $100 \mathrm{~mm}$ desde el proceso estiloides radial, mientras que el músculo L4 lo hizo entre los 57 a $90 \mathrm{~mm}$.

Si bien estas cifras son similares solamente en las distancias mínimas descritas en estos dos estudios, se visualiza que la ubicación de los diversos puntos motores es altamente variable. En la busqueda de otras metodologías que detallen la ubicación de los puntos motores, Hughes \& Clarke, determinaron que el Pm del músculo L3 estuvo en el tercio medio del tercer metacarpiano, mientras que en el caso del músculo L4 este punto estuvo en el tercio distal del cuarto metacarpiano. Si bien su metodología difiere de la nuestra, coincidimos con los puntos motores informados; siendo para ellos $68 \mathrm{~mm}$ y $66 \mathrm{~mm}$ para los músculos L3 y L4 respectivamente, versus los nuestros de 69,87 y 69,21 $\mathrm{mm}$ para los mismos músculos. Consideramos que la metodología que se adapta a las diferencias antropométricas de los sujetos es aquella que informa de los Pm en relación a los tercios de los diferentes vientres musculares. En este sentido, los músculos L1 y L2 presentaron mayoritariamente sus Pm en el tercio medio (79\% y $71 \%$, respectivamente). Para el caso de los músculos L3 y L4, los Pm se ubicaron preferentemente en sus tercios distales, alcanzando un $87 \%$ y un $83 \%$, respectivamente.

Todos estos hallazgos resultan importantes a la hora de planificar una cirugía reconstructiva de la mano. Para los cirujanos especializados en esta zona, el conocimiento de las posibles variaciones determinarán el éxito operatorio; más aún, si se requiere realizar procedimientos quirúrgicos que involucren neurolisis internas o neurorrafias de estos elementos nerviosos.

AGRADECIMIENTOS: Se agradece al ilustrador Sr. Alfredo Torres Parra por sus contribuciones gráficas a esta comunicación científica.

VEGA, C.; RIVEROS, A. \& OLAVE, E. Biometric study of the innervation of the lumbrical muscles of the human hand. Int. J. Morphol., 38(4):1096-1105, 2020.

SUMMARY: The lumbrical muscles (LM) of the human hand are key in proprioception of flexion and finger extension. The description of its innervation indicates that the median nerve (MN) innervates the two lateral LMs (L1 and L2) and the ulnar nerve (UN) the medial LMs (L3 and L4). Various authors have reported a great variability of this innervation, both in which nerve delivers branches for these muscles, as well as in the distribution of their branches and the presence of common trunks. On the other hand, the number of branches that each LM receives and the motor points $(\mathrm{Mp})$ of these have been scarcely reported. The aim of this study was to determine the number, location and $\mathrm{Mp}$ of the branches destined for the LM of the human hand. Likewise, the most frequent innervation pattern was established. For this, 24 formalized hands, belonging to the anatomy laboratory, of the Universidad Andrés Bello, Viña del Mar, Chile, were used. Conventional depth plane dissection was performed. In all cases, the branch of the L1 muscle originated from the palmar digital nerve proper to the index finger, in the same way, in $100 \%$ the L2 was supplied with a branch of the common palmar digital nerve from the second interosseous space. In relation to the LM, in $100 \%$ both muscles were innervated by branches of the deep branch of the UN (DBUN). In the case of L3, $92 \%$ presented a common trunk with the second palmar interosseous muscle. Likewise, in $79 \%$ of the cases, there was a 
common trunk between the L4 and the third palmar interosseous muscle. In $29 \%$, the L3 presented a dual innervation. The distance between of the Mp-BEstL was $63.96 \mathrm{~mm}$ for L1, $67.91 \mathrm{~mm}$ for L2 and $68.69 \mathrm{~mm}$ for L3. This distance was $69.87 \mathrm{~mm}$ for L3 and 69, $21 \mathrm{~mm}$ for $\mathrm{L} 4$. The results obtained contribute to the anatomical knowledge of the innervation of the lumbrical muscles and is useful in neurosurgery procedures that seek to restore the functionality of the hand.

KEY WORDS: Anatomy; Innervation; Lumbrical Muscle; Median Nerve; Deep Branch of the Ulnar Nerve; Communicating branch; Anatomical Variations.

\section{REFERENCIAS BIBLIOGRÁFICAS}

Afroze, M. K. H; Aswinpraksh; Ramesh, P.; Vaithianathan, G.; Balaji, T. K. \& Judson, J. P. Variation in innervation of lumbricals of hands. Int. J. Innov. Res. Dev., 3(5):243-6, 2014.

Atkins, S. E.; Logan, B. \& McGrouther, D. A. The deep (motor) branch of the ulnar nerve: a detailed examination of its course and the clinical significance of its damage. J. Hand Surg. Eur. Vol., 34(1):47-57, 2009.

Ferrari, G. P. \& Gilbert, A. The superficial anastomosis on the palm of the hand between the ulnar and median nerves. J. Hand Surg. Br., 16(5):5114, 1991.

Gil, Y. C.; Shin, K. J.; Lee, S. H.; Koh, K. S. \& Song, W. C. Anatomy of the deep branch of the ulnar nerve. J. Hand Surg. Eur. Vol., 41(8):843-7, 2016.

Homma, T. \& Sakai, T. Thenar and hypothenar muscles and their innervation by the ulnar and median nerves in the human hand. Acta Anat. (Basel), 145(1):44-9, 1992.

Hughes, L. A. \& Clarke, H. M. Normal arborization of the deep branch of the ulnar nerve into the interossei and lumbricals. J. Hand Surg. Am., 20(1):10-4, 1995

Hur, M. S. Variations of lumbrical muscle innervation patterns in the hand, focusing on the dual innervation of the third lumbrical muscle. Muscle Nerve, 55(2):160-5, 2017.

Kaplan, E. Anatomía Funcional y Quirúrgica de la Mano. Buenos Aires, Artecnica, 1961.

Lauritzen, R.; Szabo, R. M. \& Lauritzen, D. B. Innervation of the lumbrical muscles. J. Hand Surg. Br, 21(1):57-8, 1996.

Llusá, M.; Palazzi, S. \& Valer, A. Anatomía Quirúrgica del Plexo Braquial y de los Nervios Periféricos de la Extremidad Superior. Buenos Aires, Médica Panamericana, 2013.

Mehta, H. J. \& Gardner, W. U. A study of lumbrical muscles in the human hand. Am. J. Anat., 109:227-38, 1961.

Olave, E. \& del Sol, M. The unar nerve distribution: innervation of the interossei, lumbricals and pollicis adductor muscles. Int. J. Morphol., 26(4):959-62, 2008.

Olave, E.; del Sol, M. \& Gabrielli, C. Disposición y relaciones del ramo comunicante entre los nervios ulnar y mediano en la mano humana. Rev. Chil. Anat, 16(2):263-9, 1998.

Palti, R. \& Vigler, M. Anatomy and function of lumbrical muscles. Hand Clin., 28(1):13-7, 2012.

Rabischong, P. Basic problems in the restoration of prehension. Ann. Chir, 25(19):927-33, 1971.

Riveros, A.; Olave, E.; Nicholson, C.; Schorwer, K. \& Torres, H. Communicating branches between the median and ulnar nerves. Proposal for inclusion in Terminologia Anatomica. Int. J. Morphol., 37(3):1192-6, 2019.

Rouvière, H. \& Delmas, A. Anatomía Humana: Descriptiva, Topográfica y Funcional. Vol. 3. 11a ed. Barcelona, Masson, 2005.
Rowntree, T. Anomalous innervation of the hand muscles. J. Bone Joint Surg. Br., 31B(4):505-10, 1949.

Standring, S. Gray's Anatomy. The Anatomical Basis of Clinical Practice. $41^{\text {a }}$ ed. Londres, Elsevier, 2016.

Sunderland, S. \& Hughes, E. S. Metrical and non-metrical features of the muscular branches of the ulnar nerve. J. Comp. Neurol., 85:113-23, 1946.

Sunderland, S. \& Ray, L. J. Metrical and non-metrical features of the muscular branches of the median nerve. J. Comp. Neurol., 85(2):191-203, 1946.

Testut, L. \& Latarjet, A. Tratado de Anatomía Humana. Vol. 3. $9^{\text {a }}$ ed. Barcelona, Salvat, 1972

Tountas, C. \& Bergman, R. Anatomics Variations of the Upper Extremity. Londres, Churchill Livingstone, 1993.

Tubbs, S.; Rizk, E.; Shoja, M.; Loukas, M.; Barbaro, N. \& Spinner, R. Nerves and Nerve Injuries. Londres, Elsevier, 2015.

Wynter, S. \& Dissabandara, L. A comprehensive review of motor innervation of the hand: variations and clinical significance. Surg. Radiol. Anat., 40(3):259-69, 2018.

Dirección para correspondencia:

Dr. Enrique Olave

Facultad de Medicina

Universidad de La Frontera

Temuco

CHILE

Email: enrique.olave@ufrontera.cl

Recibido : 22-01-2020

Aceptado: 16-03-2020 